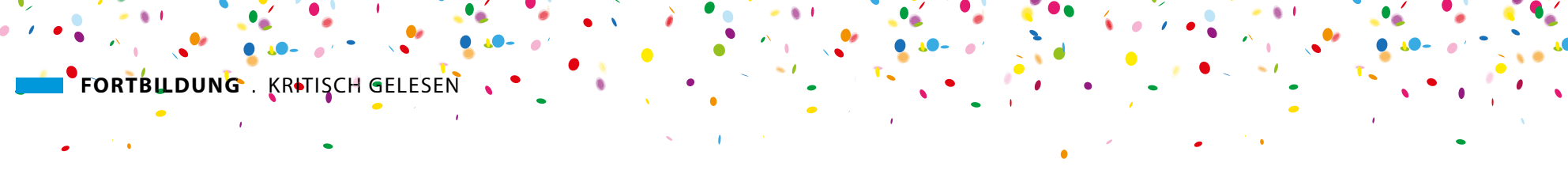

\title{
Sind Chefkardiologen völlig überflüssig?
}

\author{
Die Versorgung im Krankenhaus scheint nicht zu leiden, wenn wegen eines Kardiologenkongresses \\ viele leitende Ärzte nicht anwesend sind. Statistisch könnte das sogar von Vorteil sein.
}

\begin{abstract}
- Gerade Kardiologen haben häufig mit Akutsituationen zu tun, doch wird auch erwartet, dass sie die wichtigsten Kongresse ihres Fachgebietes besuchen. Eine retrospektive Registerstudie in den USA untersuchte nun, ob sich dieses Dilemma auf die 30-Tage-Mortalität von über 100.000 Medicare-Patienten ausgewirkt hat. Sie waren zwischen 2002 und 2011 während eines Herzkongresses wegen akutem Koronarsyndrom, Herzstillstand oder Herzinsuffizienz stationär aufgenommen worden. Als Vergleichskollektiv dienten Patienten, die in der kongressfreien Zeit aufgenommen wurden.
\end{abstract}

In akademischen Lehrkrankenhäusern war die 30-Tage-Mortalität bei den
Hochrisikopatienten mit Herzinsuffizienz $(17,5 \%$ vs. $24,8 \%$; p < 0,001) oder Herzstillstand (59,1\% vs. $69,4 \%$; $p<0,01)$ während eines Kongresses signifikant geringer als in der kongressfreien Zeit. Bei Hochrisikopatienten mit akutem Koronarsyndrom und bei allen Patienten mit niedrigem Risiko ergaben sich diesbezüglich keine Unterschiede.

- Jena AB et al. Mortality and treatment patterns among patients hospitalized with acute cardiovascular conditions during dates of national cardiology meetings. JAMA Intern Med. 2015;175:237-44

\section{KOMMENTAR}

Die Ergebnisse werfen mehr Fragen auf, als sie Antworten geben - sind aber Wasser auf die Mühlen der notorischen Kritiker, für die der Laden besser läuft, wenn der Chef nicht da ist. Andererseits könnte es durchaus sein, dass man riskante Eingriffe eben nicht in dieser Zeit einplant. Grundsätzlich erhebt sich die Frage, wie intensiv die leitenden Ärzte, die man auf Kongressen trifft, überhaupt in die unmittelbare Patientenversorgung einbezogen sind. All diese Fragen kann die Studie nicht beantworten. Kausale Attribuierungen sind schon gar nicht zu machen. Patienten zumindest können sicher sein, dass die Versorgung während großer Ärztekongresse nicht gefährdet ist. Und Chefärzte können die Studie ihrem Verwaltungsleiter zeigen, wenn der ihnen wegen längerer Abwesenheiten Vorhaltungen macht.

Prof. Dr. med. H. S. FüeßI

\section{Reden wir die Welt wenigstens schön!}

Der Euphemismus hat in unseren Zeiten offensichtlich Hochkonjunktur. Aus der Mülldeponie wird der Entsorgungspark, Schulden werden zum Wertanpassungsbedarf und das Altenheim zur Seniorenresidenz. Da darf die Medizin nicht nachstehen, gibt es doch gerade hier viele unangenehme Wahrheiten, die auf eine angenehme Verbalverpackung warten.

Die "Neubildung" für das Karzinom hat eine lange Tradition, doch wie steht es mit dem medizinischen Fortschritt? Wissenschaftler aus Australien haben hier einige nützliche Vorschläge gemacht. Angesichts hoher Sectio-Zahlen wird die natürliche Geburt zunehmend unnatürlich und sollte schnellstens umbenannt werden in "Geburt durch die obsolete uterine Austrittsmethode". Das Down-Syndrom hört sich zu negativ-abwärtsgewandt an und sollte in Zukunft als Up-Syndrom bezeichnet werden. Für Anhänger der Komplementärmedizin, die ihre Kinder nicht impfen lassen, empfiehlt sich die Bezeichnung „Euphemystiker“. Warum einem Patienten mit Herzinsuffizienz erklären, dass sein Herz nicht genügend pumpt und sich Flüssigkeit in die Lungen zurückstaut? Man könnte doch von einer vorübergehend reduzierten Arbeitskapazität des Herzmuskels sprechen. Dick ist heute ohnehin niemand mehr, sondern allenfalls metabolisch herausgefordert oder wohlgerundet gesund.

Zum größten Problem der Medizin, dem Lebensende - früher auch Tod genannt - liegen schon seit langer Zeit Vorschläge von Monty Python vor, etwa die "Wiederbegegnung mit dem Schöpfer" oder die "historische Betrachtung metabolischer Prozesse". Allerdings sind diese Termini nicht ausreichend wissenschaftlich-medizinisch. Angehörigen könnte etwa mitgeteilt werden: „Ihre Frau hat Raumtemperatur angenommen." Der Ausdruck "Palliativmedizin“ wiederum ist mittlerweile fast volkstümlich ge- worden und sollte schleunigst durch die deutlich empathischere "Neuorientierung bei der Lebensreise-Betreuung " ersetzt werden. Und was gäbe es für einen Umweltschützer Schöneres als die Mitteilung des Transplantationschirurgen, er habe „erhöhtes Recycling-Potenzial"?

Prof. Dr. med. H. S. FüeßI

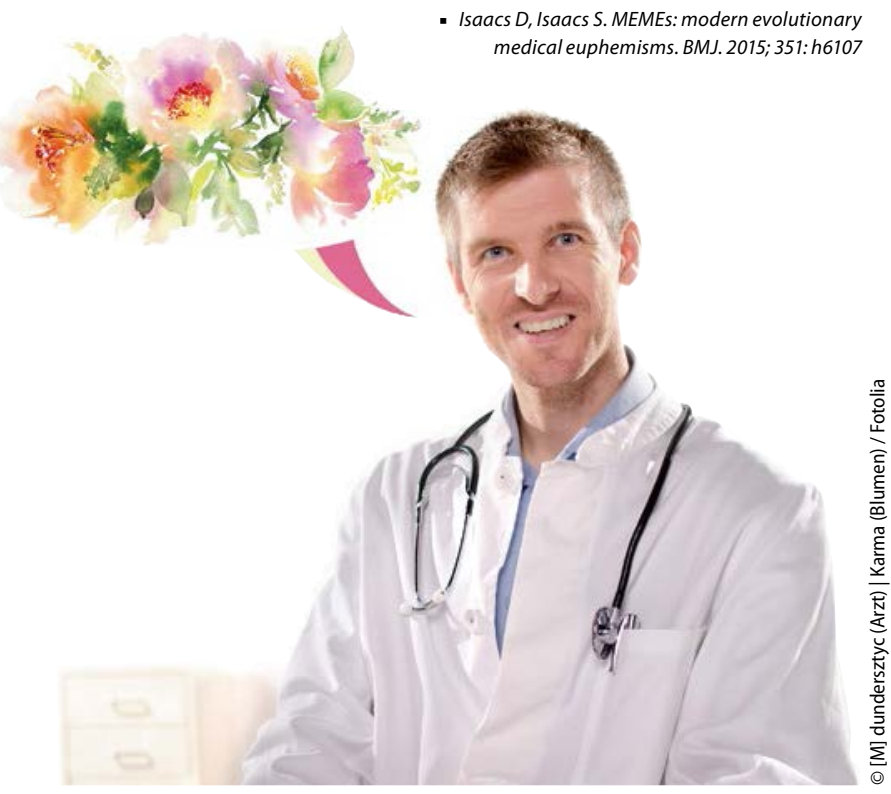

